九州大学学術情報リポジトリ

Kyushu University Institutional Repository

\title{
A SEQUENTIAL SAMPLING PLAN FOR A LOT WITH CLASSIFIED DEFECTIVES
}

Yabuuchi, Hidekazu

Department of Mathematical Sciences, College of Engineering, University of Osaka Prefecture

Sato, Masako

Department of Mathematics and Related fields, College of Integrated Arts and Sciences, University of Osaka Prefecture

https://doi.org/10.5109/13390

出版情報: Bulletin of informatics and cybernetics. 22 (3/4)，pp.225-237，1987-03. Research Association of Statistical Sciences

バージョン :

権利関係 : 


\title{
A SEQUENTIAL SAMPLING PLAN FOR A LOT WITH CLASSIFIED DEFECTIVES
}

By

\section{Hidekazu YABUUCHI* and Masako SATo**}

\begin{abstract}
We deal with a lot consisting of effectives, and defectives classified into several categories according to their inferiorities. A sequential sampling plan for the lot based on the sequential probability ratio test proposed by Wald [7] is investigated.

The primary object is to develop exact formulae for operating characteristic, average sample size and the moment generating function of the number of observations for the sequential plan. For this purpose, we use the combinatorial method for counting all the possible sampling processes falled into acceptance or rejection region. Furthermore, we propose a way of determinating constants which involve two equalities giving decision boundaries of acceptance or rejection of the lot.
\end{abstract}

\section{Introduction}

Let us consider lots containing a large number of units (products). Each unit in a lot is classified as effective or detective and furthermore, each of detectives is classified into either one of $k$ categories according to its degree of inferiority such as minor defective, major detective and so on, where $k$ is a positive integer. Suppose that $C_{0}$ is the category of effectives and $C_{i}(i=1, \cdots, k)$ is that of defectives with the $i$-th degree of inferiority. Let $p_{i}$ be the proportion of units belonging to $C_{i}$ in the lot for $i=0, \cdots, k$, where $\sum_{i=0}^{k} p_{i}=1$.

Let us consider sampling inspections for the lot satisfying the conditions that the probability of rejecting the lot does not exceed $\alpha$ whenever $p_{i} \leqq p_{0 i}$ for $i=1, \cdots, k$ and that of accepting the lot does not exceed $\beta$ whenever $p_{i} \geqq p_{1 i}$ for $i=1, \cdots, k$, where $\alpha$ is producer's risk, $\beta$ is a cosumer's risk, and $\left\{p_{0 i}\right\}$ and $\left\{p_{1 i}\right\}$ are preassigned probability distributions such that

$$
0<p_{0 i}<p_{1 i} \text { for } i=1, \cdots, k \text { and } \min _{1 \leqq i \leqq k} \frac{p_{1 i}}{p_{0 i}} \geqq \frac{p_{00}}{p_{10}} .
$$

Such sampling inspections for the lot occur often in practice but analysis for in-

* Department of Mathematical Sciences, College of Engineering, University of Osaka Prefecture, Mozu, Sakai, Osaka 591, Japan.

** Department of Mathematics and Related fields, College of Integrated Arts and Sciences, University of Osaka Prefecture, Mozu, Sakai, Osaka 591, Japan. 
spection plans having desired Operating Characteristics are not yet sufficiently developed, except for cases of $k=1$ (see for instance, [1] [3], [7]) and $k=2([6]$ ).

In this paper, we consider a sequential sampling plan based on a sequential probability ratio test proposed by $\mathrm{A}$. Wald [7].

Firstly, we represent a sequential sampling process as a lattice path in $(k+1)$ dimensional space, so that decision boundaries of acceptance or rejection of the lot can be given by two parallel hyperplanes in the space. It turns out that the probability distribution of the number of observations required by the sequential plan can be obtained by a combinatorial method of counting all the possible lattice paths falled into acceptance or rejection region.

On the other hand, Sato (one of the present authors) and Sado [4] have delt with the number of lattice paths restricted by two parallel hyperplanes in $(k+1)$-dimensional space and given explicit formulae for the generating functions of those numbers, provided that coefficients (inclines) of hyperplanes are all integral. In terms of the results, we give explicit formulae for various characteristics such as the OC, the ASN and the moment generating functions, and the deviation of the number of observations. Furthermore we propose a method for determinating the values of coefficients of the hyperplanes and illustrate the method with an example. In the example, the values of ASN can be saved probably $50 \%$ comparing with the sample size for the corresponding single sampling plan.

For a special case of $k=1$, the theoretical and numerical results are given in the other paper [5].

\section{Sequential probability ratio test}

The sequential sampling plan based on the probability ratio test satisfying the condition described in the previous section is given as follows: at $t$-th trial for any integral value $t$, let $n_{i}(i=1, \cdots, k)$ be the number of units belonging to $C_{i}$ in sample inspected successively, where $\sum_{i=1}^{k} n_{i}=t$. Then

$$
\begin{array}{ll}
\prod_{i=0}^{k}\left(p_{1 i} / p_{0 i}\right)^{n_{i}}<C & \text { (The lot is accepted) } \\
C \leqq \prod_{i=0}^{k}\left(p_{1 i} / p_{0 i}\right)^{n_{i}} \leqq B & \text { (Inspection is continued) } \\
\prod_{i=0}^{k}\left(p_{1 i} / p_{0 i}\right)^{n_{i}}>B & \text { (The lot is rejected) }
\end{array}
$$

where constants $B$ and $C(0<C<B)$ are determined to be such that the probabilites errors of the first and second kinds will take the values $\alpha$ and $\beta$.

The above inequalities can be rewritten as follows:

$$
\begin{array}{ll}
n_{0}>\sum_{i=1}^{k} d_{i} n_{i}+c & \text { (The lot is accepted) } \\
\sum_{i=1}^{k} d_{i} n_{i}-b \leqq n_{0} \leqq \sum_{i=1}^{k} d_{i} n_{i}+c \quad \text { (Inspection is continued) }
\end{array}
$$




$$
n_{0}<\sum_{i=1}^{k} d_{i} n_{i}-b \quad \text { (The lot is rejected) }
$$

where

$$
\begin{aligned}
& d_{i}=\frac{\log p_{1 i} / p_{0 i}}{\log p_{00} / p_{10}}, \quad(i=1, \cdots, k) \\
& b=\frac{\log B}{\log p_{00} / p_{10}}, \quad \text { and } \quad c=\frac{\log 1 / C}{\log p_{00} / p_{10}} .
\end{aligned}
$$

Note that the equalities in (2.2) are contained in (2.1) and (2.3) in the procedure described by Wald [7].

The parameters $\left\{d_{i}\right\}$ are determined only by the preassigned probability distributions $\left\{p_{0 i}\right\}$ and $\left\{p_{1 i}\right\}$ as shown in (2.4). However, concerning with the parameters $b$ and $c$, the explicit formula for the $\mathrm{OC}$ function is required to be evaluated the exact values of them.

\section{Lattice Path Representation of a Sampling Process}

We denote by $\left\{z_{1}, \cdots, z_{k}, z_{0}\right\}$ the set of axes in $(k+1)$-dimensional space. A (minimal) lattice path in the orthant of the space, abbreviated by LP, means a path passing through lattice points and moving one unit to positive direction along either one of $(k+1)$-axes.

Let us consider one to one correspondence between a LP and a sampling process as follows: let be correspond an occurence of unit belonging to $C_{i}$ in the sampling process to a movement of one unit to the positive direction along an axis $z_{i}$, where the initial point of LP is assumed to be origin. Thus a sampling process can be presented graphically as a LP starting from the origin. The criteria boundaries of the sampling plan given by (2.1)-(2.3) can be given by the following two parallel hyperplanes:

$$
\left\{\begin{array}{l}
h_{1}: z_{0}=\sum_{i=1}^{k} d_{i} z_{i}-b \\
h_{2}: z_{0}=\sum_{i=1}^{k} d_{i} z_{i}+c
\end{array}\right.
$$

That is, the inspection is continued as long as the corresponding LP lies between two hyperplanes $h_{1}$ and $h_{2}$, and is terminated the first time that the path crosses either one of both hyperplanes. If the path crosses $h_{2}\left(h_{1}\right)$, then the lot is accepted (rejected) (See Fig. 1). Let be denote by $\left(\boldsymbol{n}, n_{0}\right)$ the co-ordinates of a lattice point in the space, where $\boldsymbol{n}=\left(n_{1}, \cdots, n_{k}\right)$. In Fig. 1 , the number $n_{0}$ of effectives is measured along the vertical axis $z_{0}$ and the numbers $\boldsymbol{n}$ of the detectives in $\left(C_{1}, \cdots, C_{k}\right)$ along the horizontal axis $z=\left(z_{1}, \cdots z_{k}\right)$, for convenience.

The parameter $d_{i}$ defined by (2.4) is a real number larger than or equal to one, because of (1.1). Hereafter, we assume that $d_{i}, i=1, \cdots, k$, are all integral. Under the assumption, we can take $b$ and $c$ as also integral without varying the criteria regions, since each LP can pass through only lattice points. 


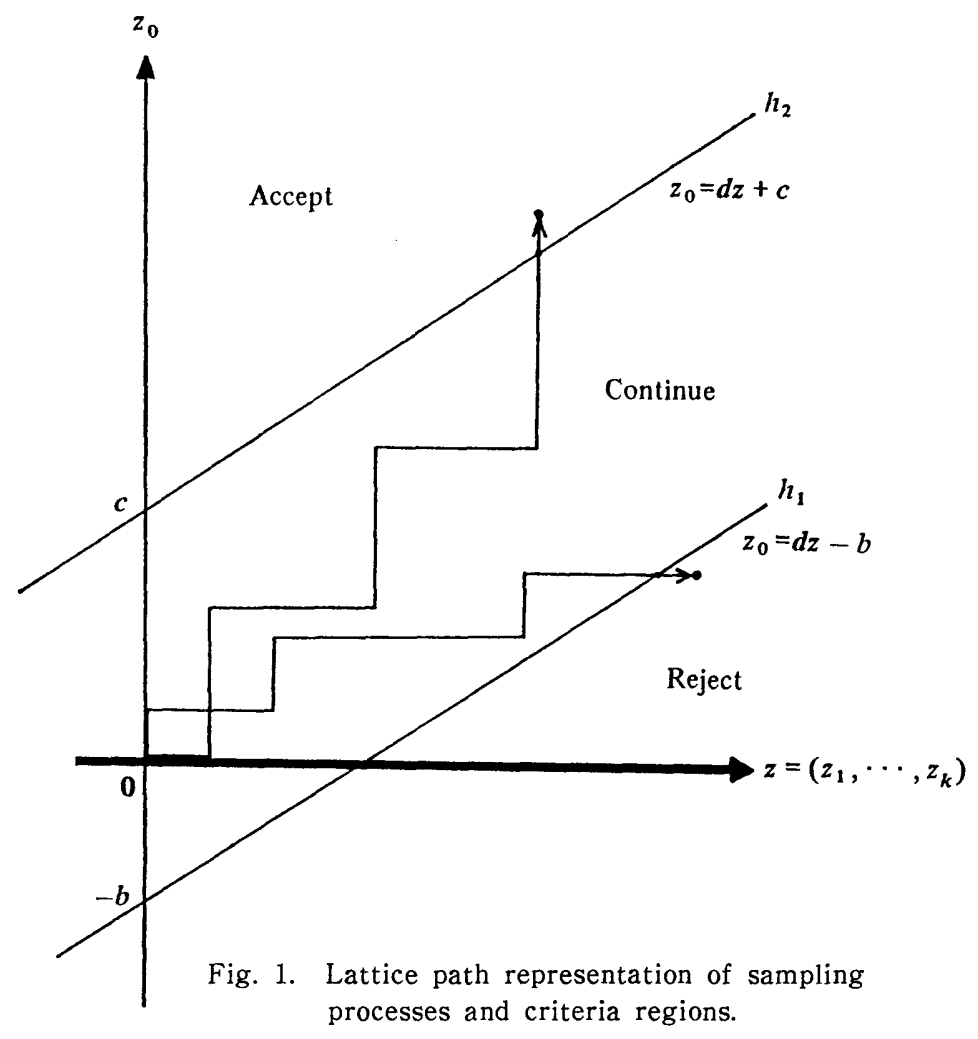

\section{OC Function}

Let be put $\boldsymbol{d}=\left(d_{1}, \cdots, d_{k}\right)$ and $\boldsymbol{p}=\left(p_{0}, \cdots, p_{k}\right)$.

For the sampling plan with parameters $(\boldsymbol{d}, b, c)$ defined by $(2.1)-(2.3)$, let us denote by $L(\boldsymbol{p} ; \boldsymbol{d}, b, c)$ the $\mathrm{OC}$ function which is the probability of accepting the lot when the true value of the proportion of $C_{i}(i=0, \cdots, k)$ in the lot is $p_{i}$. Also we denote by $\bar{L}(\boldsymbol{p} ; \boldsymbol{d}, b, c)$ the probability of rejecting the lot when the true proportion of $C_{i}(i=0$, $\cdots, k)$ is $p_{i}$. We show that these probabilities can be evaluated by counting LP's crossing either one of hyperplanes, respectively.

For non-negative integers $a, b$ and $c$, let denote by $T(\boldsymbol{n}, a, b, c)$ the number of LP's from the origin to a lattice point $(\boldsymbol{n}, \boldsymbol{d n}+a-b)$ without crossing any of $h_{1}$ and $h_{2}$ where $\boldsymbol{d} \boldsymbol{n}=\sum_{i=1}^{k} d_{i} n_{i}$, and introduce the generating function of these numbers :

$$
T(\boldsymbol{x}, a, b, c)=\sum_{\substack{\boldsymbol{d} \boldsymbol{n}+a \geq b \\ n \geq 0}} T(\boldsymbol{n}, a, b, c) \boldsymbol{x}^{n},
$$

where $\boldsymbol{x}^{n}=x_{1}^{n_{1}} \cdots x_{k}^{n_{k}}$ for $\boldsymbol{x}=\left(x_{1}, \cdots, x_{k}\right)$ and $\boldsymbol{n}=\left(n_{1}, \cdots, n_{k}\right)$ and the sum is extending over all non-negative $n_{i}(i=1, \cdots, k)$ such that $\boldsymbol{d n}+a \geqq b$. In a previous paper [4], the following expression for $T(\boldsymbol{x}, a, b, c)$ was given:

$$
T(\boldsymbol{x}, a, b, c)=\frac{\varphi(\boldsymbol{x}, b) \varphi(\boldsymbol{x}, b+c-a)}{\varphi(\boldsymbol{x}, b+c+1)}-\varphi(\boldsymbol{x}, b-a-1),
$$


where $\varphi(\boldsymbol{x}, n)$ is a polynomial of $x_{1}, \cdots, x_{k}$ such that

$$
\varphi(\boldsymbol{x}, n)=\left\{\begin{array}{cc}
\sum_{0 \leq d \boldsymbol{l} \leq n}\left(\begin{array}{c}
n-\boldsymbol{d} \boldsymbol{l} \\
\boldsymbol{l}
\end{array}\right)(-\boldsymbol{x})^{\boldsymbol{l}}, & n \geqq 0 \\
0, & n<0
\end{array}\right.
$$

where $(-\boldsymbol{x})^{l}=\left(-x_{1}\right)^{l_{1}} \cdots\left(-x_{k}\right)^{l_{k}}$ for $l=\left(l_{1}, \cdots, l_{k}\right)$, and $\left(\begin{array}{l}n \\ l\end{array}\right)$ means the multinomial coefficient.

In terms of the above result, we have the following two Theorems.

THEOREM 1.

where

$$
L(\boldsymbol{p} ; \boldsymbol{d}, b, c)=p_{0}^{c+1} \psi\left(\boldsymbol{x}_{\boldsymbol{p}}, b, c\right)
$$

and

$$
\phi(\boldsymbol{x}, b, c)=\frac{\varphi(\boldsymbol{x}, b)}{\varphi(\boldsymbol{x}, b+c+1)}
$$

$$
\boldsymbol{x}_{\boldsymbol{p}}=\left(p_{1} p_{0}^{d_{1}}, \cdots, p_{k} p_{0}^{d_{k}}\right)
$$

for $\boldsymbol{p}=\left(p_{0}, \cdots, p_{k}\right)$.

Proof. Let us consider all the possible sampling processes terminated with the acceptance of the lot, in each of which deffectives belonging to $C_{i}$ occur $n_{i}$ times for $i=1, \cdots, k$. Each of these processes corresponds to each of LP's starting from the origin to $(\boldsymbol{n}, \boldsymbol{d} \boldsymbol{n}+c)$ without crossing any of two hyperplanes and moving to $(\boldsymbol{n}, \boldsymbol{d} \boldsymbol{n}+\boldsymbol{c}$ $+1)$ after crossing the hyperplane $h_{2}$. The number of such LP's is given by $T(\boldsymbol{n}, b$ $+c, b, c)$. Since the probability of each of these LP's is given by $\left(\prod_{r=1}^{k} p_{r}^{n_{r}}\right) p_{0}^{d n+c+1}$, the probability of acceptance of the lot for processes considered is given by

$$
T(\boldsymbol{n}, b+c, b, c)\left(\prod_{r=1}^{k} p_{r}^{n_{r}}\right) p_{0}^{d n+c+1}
$$

which implies from (4.1),

$$
\begin{aligned}
L(\boldsymbol{p} ; \boldsymbol{d}, b, c) & =\sum_{n \geq 0} T(\boldsymbol{n}, b+c, b, c)\left(\prod_{r=1}^{k} p_{0}^{n_{r}}\right) p_{0}^{d n+c+1} \\
& =p_{0}^{c+1} T\left(\boldsymbol{x}_{\boldsymbol{p}}, b+c, b, c\right)=p_{0}^{c+1} \psi\left(\boldsymbol{x}_{\boldsymbol{p}}, b, c\right) .
\end{aligned}
$$

If $p_{i}, i=1, \cdots, k$, are sufficiently small, $L(\boldsymbol{p} ; \boldsymbol{d}, b, c)$ is approximately given from (4.3) by the expansion of the rational function $\psi(\boldsymbol{x}, b, c)$ as follows:

COROLLARY 1.

$$
L(\boldsymbol{p} ; \boldsymbol{d}, b, c) \sim p_{0}^{c+1}\left\{1+(c+1) \sum_{i=1}^{k} p_{i} p_{0}^{d_{i}}\right\}
$$

THEOREM 2.

$$
\bar{L}(\boldsymbol{p} ; \boldsymbol{d}, b, c)=p_{0}^{-b}\left[R\left(\boldsymbol{p}, \boldsymbol{x}_{\boldsymbol{p}}, b\right)-R\left(\boldsymbol{p}, \boldsymbol{x}_{\boldsymbol{p}}, b+c+1\right) \psi\left(\boldsymbol{x}_{\boldsymbol{p}}, b, c\right)\right]
$$

where $\phi(\boldsymbol{x}, b, c)$ and $\boldsymbol{x}_{\boldsymbol{p}}$ are defined by (4.4) and (4.5), and

$$
R(\boldsymbol{p}, \boldsymbol{x}, n)=\varphi(\boldsymbol{x}, n)-\sum_{i=1}^{k} p_{i} \sum_{j=0}^{d i-1} p_{0}^{j} \varphi(\boldsymbol{x}, n-1-j)
$$

for $\boldsymbol{p}=\left(p_{0}, \cdots, p_{k}\right)$. 
Proof. Similarly to the proof of Theorem 1, the probability of rejecting the lot by occurences of $n_{i}(i=1, \cdots, k)$ defectives belonging to $C_{i}$ is given as

$$
\sum_{i=1}^{k} p_{i} \sum_{j=0}^{d_{i-1}} T(\boldsymbol{n}, j, b, c)\left(\prod_{r=1}^{k} p_{r}^{n_{r}}\right) p_{0}^{d n-b+j}
$$

Therefore we have using (4.1)

$$
\begin{aligned}
\bar{L}(\boldsymbol{p} ; \boldsymbol{d}, b, c)= & \sum_{i=1}^{k} p_{i} \sum_{j=0}^{d i-1} \sum_{\boldsymbol{d}+j \geq b} T(\boldsymbol{n}, j, b, c)\left(\prod_{r=1}^{k} p_{r}^{n_{r}}\right) p_{0}^{d n-b+j} \\
& =\sum_{i=1}^{k} p_{i} \sum_{j=0}^{d_{i}-1} p_{0}^{j}\left[\frac{\varphi\left(\boldsymbol{x}_{\boldsymbol{p}}, b\right)}{\varphi\left(\boldsymbol{x}_{\boldsymbol{p}}, b+c+1\right)} \varphi\left(\boldsymbol{x}_{\boldsymbol{p}}, b+c-j\right)-\varphi\left(\boldsymbol{x}_{\boldsymbol{p}}, b-j-1\right)\right],
\end{aligned}
$$

which implies (4.8) from (4.9).

\section{The Properties of Polynomial $\varphi(\boldsymbol{x}, n)$ and $R(\boldsymbol{p}, \boldsymbol{x}, n)$}

In this paragraph, we discuss about equations concerning with the polynomial $\varphi(\boldsymbol{x}, n)$ or $R(\boldsymbol{p}, \boldsymbol{x}, n)$, which are used later in this paper.

Firstly, we give the generating function for $\{\varphi(\boldsymbol{x}, n), n \geqq 0\}$ and a difference equation of $\varphi(\boldsymbol{x}, n)$ which is useful for numerical calculations of $\varphi\left(\boldsymbol{x}_{\boldsymbol{p}}, n\right)$.

LEMMA 1.

$$
\sum_{n=0}^{\infty} \varphi(\boldsymbol{x}, n) z^{n}=\frac{1}{A(\boldsymbol{x}, z)},
$$

where

$$
A(\boldsymbol{x}, z)=1-z+\sum_{i=1}^{k} x_{i} z^{d_{i+1}}
$$

for $\boldsymbol{x}=\left(x_{1}, \cdots, x_{k}\right)$.

(ii) The following difference equation is valid:

$$
\varphi(\boldsymbol{x}, n)= \begin{cases}1, & n=0 \\ \varphi(\boldsymbol{x}, n-1)-\sum_{i=1}^{k} x_{i} \varphi\left(\boldsymbol{x}, n-d_{i}-1\right), & n \geqq 1 .\end{cases}
$$

Proof. (i) From the definition of $\varphi(\boldsymbol{x}, n)$, we have

$$
\begin{aligned}
& \sum_{n=0}^{\infty} \varphi(\boldsymbol{x}, n) z^{n}=\sum_{n=0}^{\infty} \sum_{\boldsymbol{l} \leq \leq n}\left(\begin{array}{c}
n-\boldsymbol{d} \boldsymbol{l} \\
\boldsymbol{l}
\end{array}\right)(-\boldsymbol{x})^{\boldsymbol{l}} z^{n}
\end{aligned}
$$

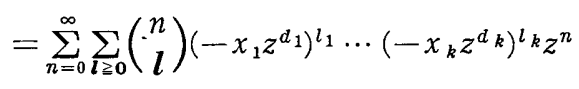

$$
\begin{aligned}
& =\sum_{n=0}^{\infty}\left(z-\sum_{i=1}^{k} x_{i} z^{d_{\imath}+1}\right)^{n},
\end{aligned}
$$

which is equal to the right hand side of (5.1).

(ii) It is clear since (i).

LEMMA 2.

$$
\sum_{j=1}^{k}\left(d_{j}+1\right) x_{j} \varphi^{(j)}(\boldsymbol{x}, n)=\varphi^{(1)}\left(\boldsymbol{x}, n+d_{1}\right)+n \varphi(\boldsymbol{x}, n)
$$


where $\varphi^{(j)}(\boldsymbol{x}, n)=\frac{\partial}{\partial x_{j}} \varphi(\boldsymbol{x}, n), j=1, \cdots, k$.

Proof. Partial differentiating the botn side of (5.1) with respect to $x_{j}(j=1, \cdots, k)$ and $z$, we have

and

$$
\sum_{n=0}^{\infty} \varphi^{(j)}(\boldsymbol{x}, n) z^{n}=\frac{-z^{d_{j}+1}}{A(\boldsymbol{x}, z)^{2}}, \quad j=1, \cdots, k
$$

which give

$$
\sum_{n=0}^{\infty} n \varphi(\boldsymbol{x}, n) z^{n}=\frac{z-\sum_{i=1}^{k}\left(d_{i}+1\right) x_{i} z^{d^{i}+1}}{A(\boldsymbol{x}, z)^{2}},
$$

$$
\sum_{n=0}^{\infty} \sum_{j=1}^{k}\left(d_{j}+1\right) x_{j} \varphi^{(j)}(\boldsymbol{x}, n) z^{n}=\sum_{n=0}^{\infty} \varphi^{(1)}\left(\boldsymbol{x}, n+d_{1}\right) z^{n-d_{1}}+\sum_{n=0}^{\infty} n \varphi(\boldsymbol{x}, n) z^{n} .
$$

Comparing with the coefficients of $z^{n}$ in the both side of the above equation, we can get (5.3).

Hereafter, we use the notation of dash(') or two-dash(") instead of the partial derivative $\frac{\partial}{\partial x_{1}}$ or $\frac{\partial^{2}}{\partial x_{1}^{2}}$.

LEMMA 3. Let $\omega$ be a real variable.

$$
\begin{aligned}
& R\left(\boldsymbol{p}_{\omega}, \boldsymbol{x}_{\boldsymbol{p}_{\omega}}, n\right)=\left(p_{0} e^{\omega}\right)^{n}+\left(1-e^{\omega}\right) S\left(\boldsymbol{p}_{\omega}, \boldsymbol{x}_{\boldsymbol{p}_{\omega}}, n-1\right) \\
& R\left(\boldsymbol{p}, \boldsymbol{x}_{\boldsymbol{p}}, n\right)=p_{0}^{n},
\end{aligned}
$$

where $\boldsymbol{x}_{\boldsymbol{p}}$ and $R(\boldsymbol{p}, \boldsymbol{x}, n)$ are defined by (4.5) and (4.9),

$$
S(\boldsymbol{p}, \boldsymbol{x}, n)=\sum_{l=0}^{n} p_{0}^{l} \varphi(\boldsymbol{x}, n-l)
$$

and

$$
\boldsymbol{p}_{\omega}=e^{\omega} \boldsymbol{p} .
$$

PROOF. (i) The generating functions for $R(\boldsymbol{p}, \boldsymbol{x}, n)$ and $S(\boldsymbol{p}, \boldsymbol{x}, n)$ defined by (4.9) and (5.7) may be derived from Lemma 1(i) as follows:

and

$$
\sum_{n=0}^{\infty} R(\boldsymbol{p}, \boldsymbol{x}, n) z^{n}=\frac{B(\boldsymbol{p}, z)}{A(\boldsymbol{x}, z)}
$$

$$
\sum_{n=0}^{\infty} S(\boldsymbol{p}, \boldsymbol{x}, n) z^{n}=\frac{1}{\left(1-p_{0} z\right) A(\boldsymbol{x}, z)},
$$

respectively, where $A(\boldsymbol{x}, n)$ is defined by (5.2) and

Since

$$
B(\boldsymbol{p}, z)=1-\sum_{i=1}^{k} p_{i} \sum_{j=0}^{d i-1} p_{0}^{j} z^{j+1} .
$$

$$
A\left(\boldsymbol{x}_{\boldsymbol{p}_{\omega}}, z\right)=\left(1-p_{0} z e^{\omega}\right) B\left(\boldsymbol{p}_{\omega}, z\right)-\left(1-e^{\omega}\right) z,
$$

it turns out from (5.9) and (5.10) that

$$
\begin{aligned}
\sum_{n=0}^{\infty} R\left(\boldsymbol{p}_{\omega}, \boldsymbol{x}_{\boldsymbol{p}_{\omega}}, n\right) z^{n} & =\frac{1}{1-p_{0} z e^{\omega}}\left\{1+\frac{\left(1-e^{\omega}\right) z}{A\left(\boldsymbol{x}_{\boldsymbol{p}_{\omega}}, z\right)}\right\} \\
& =\frac{1}{1-p_{0} z e^{\omega}}+\left(1-e^{\omega}\right) \sum_{n=0}^{\infty} S\left(\boldsymbol{x}_{\boldsymbol{p}_{\omega}}, n\right) z^{n+1} .
\end{aligned}
$$


Comparing with coefficients of $z^{n}$ in the both side of the above equation, we get (5.5).

(ii) Since (5.5) for $\omega=0,(5.6)$ is valid.

LEMMA 4.

(i) $S\left(\boldsymbol{p}, \boldsymbol{x}_{\boldsymbol{p}}, n\right)=-R^{\prime}\left(\boldsymbol{p}, \boldsymbol{x}_{\boldsymbol{p}}, n+d_{1}+1\right)$

(ii) $\left[\frac{\partial}{\partial \omega} S\left(\boldsymbol{p}_{\omega}, \boldsymbol{x}_{\boldsymbol{p}_{\omega}}, n\right)\right]_{\omega=0}=-\frac{1}{2}\left[R^{\prime \prime}\left(\boldsymbol{p}, \boldsymbol{x}_{\boldsymbol{p}}, n+2 d_{1}+1\right)+2 n R^{\prime}\left(\boldsymbol{p}, \boldsymbol{x}_{\boldsymbol{p}}, n+d_{1}+1\right)\right]$

where $\boldsymbol{x}_{p}, R(\boldsymbol{p}, \boldsymbol{x}, n), S(\boldsymbol{p}, \boldsymbol{x}, n)$ and $\boldsymbol{p}_{\omega}$ are defined by (4.5), (4.9), (5.7) and (5.8).

PROOF. (i) From (5.12) for $\omega=0$, it follows that

$$
A\left(\boldsymbol{x}_{p}, z\right)=\left(1-p_{0} z\right) B(\boldsymbol{p}, z) .
$$

After partial differentiating the both side of (5.9) w.r.t. $x_{1}$ we put $\boldsymbol{x}=\boldsymbol{x}_{\boldsymbol{p}}$, it turns out (5.10) and (5.15) that

$$
\begin{aligned}
\sum_{n=0}^{\infty} R^{\prime}\left(\boldsymbol{p}, \boldsymbol{x}_{\boldsymbol{p}}, n\right) z^{n} & =\frac{-B(\boldsymbol{p}, z) z^{d_{1}+1}}{A\left(\boldsymbol{x}_{\boldsymbol{p}}, z\right)^{2}}=\frac{-z^{d_{1}+1}}{\left(1-p_{0} z\right) A\left(\boldsymbol{x}_{\boldsymbol{p}}, z\right)} \\
& =-z^{d_{1}+1} \sum_{n=1}^{\infty} S\left(\boldsymbol{p}, \boldsymbol{x}_{\boldsymbol{p}}, n\right) z^{n},
\end{aligned}
$$

which gives (5.12).

(ii) Similarly to (i), it follows that

$$
\sum_{n=0}^{\infty} R^{\prime \prime}\left(\boldsymbol{p}, \boldsymbol{x}_{\boldsymbol{p}}, n\right) z^{n}=\frac{z^{2\left(d_{1}+1\right)}}{2\left(1-p_{0} z\right) A\left(\boldsymbol{x}_{\boldsymbol{p}}, z\right)^{2}} .
$$

Hence we have from (5.10)

$$
\begin{aligned}
{\left[\frac{\partial}{\partial \omega} \sum_{n=0}^{\infty} S\left(\boldsymbol{p}_{\omega}, \boldsymbol{x}_{\boldsymbol{p}_{\omega}}, n\right) z^{n}\right]_{\omega=0} } & =z\left(\frac{\partial}{\partial z} \sum_{n=0}^{\infty} S\left(\boldsymbol{p}, \boldsymbol{x}_{\boldsymbol{p}}, n\right) z^{n}\right)-\frac{z}{\left(1-p_{0} z\right) A\left(\boldsymbol{x}_{\boldsymbol{p}}, z\right)^{2}} \\
& =\sum_{n=0}^{\infty} n S\left(\boldsymbol{p}, \boldsymbol{x}_{\boldsymbol{p}}, n\right) z^{n}-\frac{1}{2} z^{-\left(2 d_{1}+1\right)} \sum_{n=0}^{\infty} R^{\prime \prime}\left(\boldsymbol{p}, \boldsymbol{x}_{\boldsymbol{p}}, n\right) z^{n},
\end{aligned}
$$

which together with (5.13), implies (5.14).

\section{The Moment Generating Function for Sample Size}

Let us denote by $T$ the number of observations required by the sequential sampling plan. Then $T$ is a random variable.

In this section, we derive a formula for the moment generating function of $T$, denoted by $E_{p}\left[e^{\omega T}\right]$.

It has been already shown that the probability that the sequential sampling procedure will eventually terminate is one ([5]). Here we show that it can be proved easily using Theorem 1, Theorem 2 and Lemma 3 (ii).

THEOREM 3.

$$
L(\boldsymbol{p} ; \boldsymbol{d}, b, c)+\bar{L}(\boldsymbol{p} ; \boldsymbol{d}, b, c)=1 .
$$

Proof. From Theorem 1, Theorem 2 and Lemma 3(ii), we have 


$$
\begin{aligned}
\bar{L}(\boldsymbol{p} ; \boldsymbol{d}, b, c) & =p_{0}^{-b}\left[p_{0}^{b}-p_{0}^{b+c+1} \cdot \psi\left(\boldsymbol{x}_{\boldsymbol{p}}, b, c\right)\right] \\
& =1-L(\boldsymbol{p} ; \boldsymbol{d}, b, c) .
\end{aligned}
$$

THEOREM 4.

(ii)

$$
\begin{aligned}
E_{\boldsymbol{p}}\left[e^{\omega T}\right]= & \left(p_{0} e^{\omega}\right)^{c+b+1} \cdot \psi\left(\boldsymbol{x}_{\boldsymbol{p}_{\omega}}, b, c\right)+\left(p_{0} e^{\omega}\right)^{-b} \\
& \cdot\left\{R\left(\boldsymbol{p}_{\omega}, \boldsymbol{x}_{\boldsymbol{p}_{\omega}}, b\right)-R\left(\boldsymbol{p}_{\omega}, \boldsymbol{x}_{\boldsymbol{p}_{\omega}}, b+c+1\right) \cdot \psi\left(\boldsymbol{x}_{\boldsymbol{p}_{\omega}}, b, c\right)\right\} \\
E_{\boldsymbol{p}}\left[e^{\omega T}\right]= & 1+\left(1-e^{\omega}\right)\left(p_{0} e^{\omega}\right)^{-b}\left[S\left(\boldsymbol{p}_{\omega}, \boldsymbol{x}_{\boldsymbol{p}_{\omega}}, b-1\right)\right. \\
& \left.-S\left(\boldsymbol{p}_{\omega}, \boldsymbol{x}_{\boldsymbol{p}_{\omega}}, b+c\right) \cdot \psi\left(\boldsymbol{x}_{\boldsymbol{p}_{\omega}}, b, c\right)\right]
\end{aligned}
$$

where $\phi(\boldsymbol{x}, b, c), \boldsymbol{x}_{\boldsymbol{p}}, R(\boldsymbol{p}, \boldsymbol{x}, n), S(\boldsymbol{p}, \boldsymbol{x}, n)$ and $\boldsymbol{p}_{\omega}$ are defined by (4.4), (4.5), (4.9), (5.7) and (5.8).

PROof. (i) Let us consider the sampling processes delt in the proof of Theorem 1 or Theorem 2. Clearly, the sample size of each of these processes is equal to the sum of powers of $p_{i}(i=0, \cdots, k)$ appearing in the expression ((4.6) or (4.10)) of the probability of its occurence, for instance that for a sampling process with probability $\left(\prod_{r=1}^{k} p_{r}^{n_{r}}\right) p_{0}^{d n+c+1}$ (see (4.6)) the size is $\sum_{r=1}^{k} n_{r}+\boldsymbol{d} \boldsymbol{n}+c+1$. It means that $E_{\boldsymbol{p}}\left[e^{\omega T}\right]$ is equal to the sum of the right hand sides of (4.3) and (4.8) substituting $p_{i} e^{\omega}$ instead of $p_{i}$ for $i=0,1, \cdots, k$. Thus we find (6.2).

(ii) From (6.2) and Lemma 3(i), (6.3) is valid.

\section{The ASN Function and the Second Moment of $T$}

In this section, we show explicit formulae for the ASN and the second moment of $T$, denoted by $E_{p}[T]$ and $E_{p}\left[T^{2}\right]$, respectively.

THEOREM 5.

$$
E_{p}[T]=p_{0}^{-b}\left[R^{\prime}\left(\boldsymbol{p}, \boldsymbol{x}_{\boldsymbol{p}}, b+d_{1}\right)-R^{\prime}\left(\boldsymbol{p}, \boldsymbol{x}_{p}, b+c+1+d_{1}\right) \cdot \psi\left(\boldsymbol{x}_{p}, b, c\right)\right],
$$

where $\phi(\boldsymbol{x}, b, c), \boldsymbol{x}_{\boldsymbol{p}}$ and $R(\boldsymbol{p}, \boldsymbol{x}, n)$ are defined by (4.4), (4.5) and (4.9).

Proof. It follows from Theorem 4 (ii) and Lemma 5 (i) that

$$
\begin{aligned}
E_{\boldsymbol{p}}[T] & =\left[\frac{\partial}{\partial \omega} E_{p}\left[e^{\omega T}\right]\right]_{\omega=0} \\
& =p_{0}^{-b}\left[S\left(\boldsymbol{p}, \boldsymbol{x}_{p}, b+c\right) \cdot \psi\left(\boldsymbol{x}_{\boldsymbol{p}}, b, c\right)-S\left(\boldsymbol{p}, \boldsymbol{x}_{\boldsymbol{p}}, b-1\right)\right] \\
& =p_{0}^{-b}\left[R^{\prime}\left(\boldsymbol{p}, \boldsymbol{x}_{\boldsymbol{p}}, b+d_{1}\right)-R^{\prime}\left(\boldsymbol{p}, \boldsymbol{x}_{\boldsymbol{p}}, b+c+1+d_{1}\right) \cdot \phi\left(\boldsymbol{x}_{\boldsymbol{p}}, b, c\right)\right] .
\end{aligned}
$$

THEOREM 6.

$$
\begin{aligned}
E_{p}\left[T^{2}\right]= & p_{0}^{-b}\left[R^{\prime \prime}\left(\boldsymbol{p}, \boldsymbol{x}_{\boldsymbol{p}}, b+2 d_{1}\right)-R^{\prime \prime}\left(\boldsymbol{p}, \boldsymbol{x}_{\boldsymbol{p}}, b+c+1+2 d_{1}\right) \cdot \psi\left(\boldsymbol{x}_{p}, b, c\right)\right. \\
& +\frac{2 R^{\prime}\left(\boldsymbol{p}, \boldsymbol{x}_{\boldsymbol{p}}, b+c+1+d_{1}\right)}{\varphi\left(\boldsymbol{x}_{\boldsymbol{p}}, b+c+1\right)}\left\{\varphi^{\prime}\left(\boldsymbol{x}_{\boldsymbol{p}}, b+c+1+d_{1}\right) \cdot \psi\left(\boldsymbol{x}_{p}, b, c\right)\right. \\
& \left.\left.-\varphi^{\prime}\left(\boldsymbol{x}_{\boldsymbol{p}}, b+d_{1}\right)\right\}\right]-E_{\boldsymbol{p}}[T],
\end{aligned}
$$

where $\varphi(\boldsymbol{x}, n), \psi(\boldsymbol{x}, b, c), \boldsymbol{x}_{\boldsymbol{p}}$ and $R(\boldsymbol{p}, \boldsymbol{x}, n)$ are defined by (4.2), (4.4), (4.5) and (4.9).

Proof. It follows from Theorem 4 (ii) and Theorem 5 that 


$$
\begin{aligned}
E_{\boldsymbol{p}}\left[T^{2}\right]= & (1-2 b) E_{\boldsymbol{p}}[T] \\
& +2 p^{-b} \frac{\partial}{\partial \omega}\left[S\left(\boldsymbol{p}_{\omega}, \boldsymbol{x}_{\boldsymbol{p}_{\omega}}, b+c\right) \cdot \psi\left(\boldsymbol{x}_{\boldsymbol{p}_{\omega}}, b, c\right)-S\left(\boldsymbol{p}_{\omega}, \boldsymbol{x}_{\boldsymbol{p}_{\omega}}, b-1\right)\right]_{\omega=0}
\end{aligned}
$$

From Lemma 5 (ii), we get

and

$$
\begin{aligned}
& {\left[\frac{\partial}{\partial \omega} S\left(\boldsymbol{p}, \boldsymbol{x}_{\boldsymbol{p}_{\omega}}, b+c\right)\right]_{\omega=0}} \\
& =-\frac{1}{2}\left[R^{\prime \prime}\left(\boldsymbol{p}, \boldsymbol{x}_{\boldsymbol{p}}, b+c+2 d_{1}+1\right)+2(b+c) R^{\prime}\left(\boldsymbol{p}, \boldsymbol{x}_{\boldsymbol{p}}, b+c+d_{1}+1\right)\right]
\end{aligned}
$$

$$
\begin{aligned}
& {\left[\frac{\partial}{\partial \omega} S\left(\boldsymbol{p}, \boldsymbol{x}_{\boldsymbol{p}_{\omega}}, b-1\right)\right]_{\omega=0}} \\
& =-\frac{1}{2}\left[R^{\prime \prime}\left(\boldsymbol{p}, \boldsymbol{x}_{p}, b+2 d_{1}\right)+2(b-1) R^{\prime}\left(\boldsymbol{p}, \boldsymbol{x}_{\boldsymbol{p}}, b+d_{1}\right)\right] .
\end{aligned}
$$

On the other hand, we have from Lemma 2

which implies

$$
\begin{aligned}
{\left[\frac{\partial}{\partial \omega} \varphi\left(\boldsymbol{x}_{\boldsymbol{p}_{\omega}}, n\right)\right]_{\omega=0} } & =\sum_{j=1}^{k}\left(d_{j}+1\right) p_{j} p_{0}^{d j} \varphi^{(j)}\left(\boldsymbol{x}_{\boldsymbol{p}}, n\right) \\
& =\varphi^{\prime}\left(\boldsymbol{x}_{\boldsymbol{p}}, n+d_{1}\right)+n \varphi\left(\boldsymbol{x}_{\boldsymbol{p}}, n\right),
\end{aligned}
$$

$$
\begin{aligned}
& {\left[\frac{\partial}{\partial \omega} \psi\left(\boldsymbol{x}_{\boldsymbol{p}_{\omega}}, b, c\right)\right]_{\omega=0}} \\
& =\frac{\varphi^{\prime}\left(\boldsymbol{x}_{p}, b+d_{1}\right)-\psi\left(\boldsymbol{x}_{\boldsymbol{p}}, b, c\right) \varphi^{\prime}\left(\boldsymbol{x}_{p}, b+c+1+d_{1}\right)}{\varphi\left(\boldsymbol{x}_{p}, b+c+1\right)}-(c+1) \psi\left(\boldsymbol{x}_{\boldsymbol{p}}, b, c\right)
\end{aligned}
$$

Substituting (7.4)-(7.6) to (7.3), we get (7.2).

\section{Determination of the Values of Parameters}

In this section, we propose a method for determinating the values of the parameters $(\boldsymbol{d}, b, c)$ and illustrate it with an example. After determinating the values of parameters, we evaluate the values of the ASN and the deviation of $T$ using the formulae obtained in the previous sections, and furthermore compare with the sample size for the corresponding single sampling plan.

In the sequential probability ratio test, the values of $d_{i}(i=1, \cdots, k)$ are determined by the preassigned probabilities $\left\{p_{0 i}\right\}$ and $\left\{p_{1 i}\right\}$ as defined by (2.4) and those of $b$ and $c$ are determined to be such that

where

$$
\alpha(\boldsymbol{d}, b, c)=\alpha \text { and } \beta(\boldsymbol{d}, b, c)=\beta,
$$

$$
\begin{aligned}
& \alpha(\boldsymbol{d}, b, c)=1-L\left(\boldsymbol{p}_{0} ; \boldsymbol{d}, b, c\right) \\
& \beta(\boldsymbol{d}, b, c)=L\left(\boldsymbol{p}_{1} ; \boldsymbol{d}, b, c\right),
\end{aligned}
$$

for $\boldsymbol{p}_{i}=\left(p_{i 0}, \cdots, p_{i k}\right)(i=0,1)$ and the preassigned values $\alpha$ and $\beta$. But, the value of $d_{i}$ given by (2.4) is not necessarily integral, and the equalities of (8.1) are not generally valid because the number of observations can take only integral values. Hence we shall determine the values of $\boldsymbol{d}, b$ and $c$ as follows: Firstly, let us introduce a set 


$$
\mathscr{D}=\left\{\boldsymbol{d}: d_{i}=\left[d_{i}^{*}\right] \text { or }\left[d_{i}^{*}\right]+1, i=1, \cdots, k\right\},
$$

where $\left[d_{i}^{*}\right]$ means the largest integer not exceeing $d_{i}^{*}$ and

$$
d_{i}^{*}=\frac{\log p_{1 i} / p_{0 i}}{\log p_{00} / p_{10}}, \quad i=1, \cdots, k .
$$

We choose the optimal $\boldsymbol{d}$ among the set $\mathscr{D}$ consisting of $2^{k}$ possible $\boldsymbol{d}$ 's, which is determined together with the values of $b$ and $c$ such that

$$
\{\alpha+\beta-\alpha(\boldsymbol{d}, b, c)-\beta(\boldsymbol{d}, b, c)\}
$$

is a minimum, under the conditions

$$
\alpha(\boldsymbol{d}, b, c) \leqq \alpha \text { and } \beta(\boldsymbol{d}, b, c) \leqq \beta .
$$

At the time finding in practice for the optimal values satisfying above condition, the following two Lemmas are useful.

LEMMA 5. (i) $L(\boldsymbol{p} ; \boldsymbol{d}, b, c)$ is a increasing function of $b$ and a decreasing function of $c$ and $d_{i}(i=1, \cdots, k)$.

(ii) $E_{p}[T]$ is a increasing function of $b$ and $c$.

PROOF. It is clear because of the consideration of the regions of acceptance or rejection of the lot.

LEMMA 6. If $d_{i}=d_{i}^{*}$ for $i=1, \cdots k$ and (8.5) are satisfied, then

is valid, where

$$
c \geqq c_{w}
$$

$$
c_{w}=\frac{\log (1-\alpha) / \beta}{\log p_{00} / p_{10}}-1
$$

Proof. If $d_{i}=d_{i}^{*}$ for $i=1, \cdots, k$, then from (4.5) we have $\boldsymbol{x}_{\boldsymbol{p}_{0}}=\boldsymbol{x}_{\boldsymbol{p}_{1}}$, which means

so that

$$
\varphi\left(\boldsymbol{x}_{p_{0}}, n\right)=\varphi\left(\boldsymbol{x}_{\boldsymbol{p}_{1}}, n\right), \quad n \geqq 0,
$$

$$
\phi\left(\boldsymbol{x}_{\boldsymbol{p}_{0}}, b, c\right)=\phi\left(\boldsymbol{x}_{\boldsymbol{p}_{1}}, b, c\right) .
$$

Consequently, it follows from Theorem 1 and (8.5) that

which give (8.6).

$$
\left(p_{00} / p_{10}\right)^{c+1} \geqq \frac{1-\alpha}{\beta} \text {. }
$$

Note that the equality of (8.6) is hold for a case of (8.1) and $c_{w}$ is the same as Wald's approximate formula for $c$ ([7]).

In practical calculation, we adopt $b_{w}$ and $c_{w}$ as the initial values of $b$ and $c$, where $b_{w}$ is Wald's approximate formula for $b$ as defined by

$$
b_{w}=\frac{\log (1-\beta) / \alpha}{\log p_{00} / p_{10}}-1,
$$

and find the optimal values of $(\boldsymbol{d}, b, c)$, taking Lemma 4 (i) into consideration.

As an illustration, consider the following example:

EXAMPLE. (A case of $k=2$ ) Let us consider a lot with three categories such that $C_{0}=\{$ effectives $\}, C_{1}=\{$ minor defectives $\}$ and $C_{2}=\{$ major defectives $\}$. Let 
Then we have

$$
\begin{array}{ll}
p_{01}=0.005, & p_{02}=0.015 \\
p_{11}=0.02, & p_{12}=0.06 \\
\alpha=0.05, & \beta=0.1
\end{array}
$$

$$
d_{1}^{*}=21.94 \quad d_{2}^{*}=21.94 \quad b_{w}=34.63 \quad c_{w}=44.75 .
$$

After numerical calculations, we find the optimal values:

where

$$
d_{1}=21 \quad d_{2}=22 \quad b=38 \quad c=35,
$$

$$
\alpha(\boldsymbol{d}, b, c)=0.0478 \quad \beta(\boldsymbol{d}, b, c)=0.0993 .
$$

For these optimal values, we get

$$
\begin{array}{ll}
E_{\boldsymbol{p}_{0}}[T]=58.968 & E_{\boldsymbol{p}_{1}}[T]=46.929 \\
D_{\boldsymbol{p}_{0}}[T]=34.883 & D_{\boldsymbol{p}_{1}}[T]=38.072,
\end{array}
$$

where $D_{p}[T]$ means the deviation of $T$.

Now we compare the above results with those for single sampling plan. Let denote $c_{i}(i=1,2)$ for the acceptance number of defectives belonging to $C_{i}$ and $t$ for the sample size. Then we have the following results:

$$
c_{1}=2 \quad c_{2}=4 \quad t=115,
$$

where the first and second kinds of errors are given and denoted by

$$
\alpha\left(t, c_{1}, c_{2}\right)=0.0499 \quad \beta\left(t, c_{1}, c_{2}\right)=0.0999 .
$$

In Table 1 , the values of the $\mathrm{OC}$ functions (denoted by $L\left(\boldsymbol{p} ; t, c_{1}, c_{2}\right)$ ) for the single sampling plans, the values of $L(\boldsymbol{p} ; \boldsymbol{d}, b, c)$ and $E_{\boldsymbol{p}}[T]$ considered above are given in the upper, middle and lower rows of arrays, respectively, for several values of $p_{1}$ and

Table 1. The values of $L\left(\boldsymbol{p} ; t, c_{1}, c_{2}\right), L(\boldsymbol{p} ; \boldsymbol{d}, b, c)$ and $E_{\boldsymbol{p}}(T)$.

\begin{tabular}{|c|c|c|c|c|c|}
\hline \multicolumn{1}{|c|}{$p_{2}$} & 0.010 & 0.015 & 0.030 & 0.045 & 0.060 \\
\hline 0.001 & .9936 & .9697 & .7363 & .4057 & .1738 \\
& .9917 & .9747 & .8128 & .5090 & .2557 \\
& 47.239 & 53.647 & 72.032 & 74.945 & 63.002 \\
\hline 0.005 & .9735 & .9501 & .7211 & .3971 & .1700 \\
& .9807 & .9522 & .7428 & .4328 & .2104 \\
& 52.191 & 58.968 & 75.035 & 72.948 & 59.416 \\
\hline 0.010 & .8855 & .8640 & .6548 & .3597 & .1535 \\
& .9552 & .9079 & .6444 & .3470 & .1641 \\
\hline 0.015 & 58.864 & 65.586 & 76.877 & 69.345 & 54.995 \\
& .7464 & .7281 & .5506 & .3012 & .1279 \\
& .9119 & .8429 & .5418 & .2743 & .1277 \\
\hline 0.020 & .55 .574 & 71.454 & 76.513 & 65.022 & 50.803 \\
\hline & .8914 & .5767 & .4349 & .2367 & .0999 \\
& 71.563 & .7582 & .4435 & .2148 & .0993 \\
\hline & & 75.764 & 74.243 & 60.430 & 46.929 \\
\hline
\end{tabular}


$p_{2}$. So far as the table shows, it may be considered that two OC curves are almost similar. It comes to the conclusion that the values of the ASN for the sequential plan can be saved as much as $50 \%$ comparing with the sample size for corresponding single sampling plan.

\section{Acknowledgement}

The authors with to express their gratitude to Dr. T. Kitagawa, the president of the International Institute for Advanced Study of Social Information Science, Fujitsu, for his suggestions and encouragement throughout.

\section{References}

[1] BartKy, W.: Multiple Sampling with Constant Probability, Annales of Math. Stat., 14 (1943), 363-377.

[2] Burmann, B. A. J.P.: Sequential Sampling Formulae for a Binomial Population, Supplement to J. Roy. Stat. Soc., (1946), 98-103.

[3] GIRshik, M.A.: Contributions to the Theory of Sequential Analysis, II, III, Annals of Math. Stat., 17 (1946), 282-298.

[4] SAto, M. and SAdo, T.: Lattice Paths Restricted by Two Parallel Hyperplanes, Bulletin of Informatics and Cybernetics, 21 (1985), 97-105.

[5] Sato, M., Yabuuchi, H. and Toyoda, M.: Sequential Sampling Formulae Derived from Lattice Path Counting, (to appear)

[6] Takahashi, H.: The Study of the Operating Characteristics for the Handicap-penalty Schemes with the Minor and Major Defectives, Bull. Facul. Sci. \& Eng. Chuo Univ., 22 (1979), 467-478.

[7] WALD, A.: Sequential analysis, John Willey \& Sons, INC. New York, (1947).

Communicated by T. Kitagawa

Received October 17, 1986 